\title{
SELF MOTIONS OF THE PANTOPTERON
}

\author{
Sébastien Briot \\ Department of Automated Manufacturing Engineering \\ École de technologie supérieure (ÉTS), Montreal, QC, Canada \\ sebastien.briot.1@ens.etsmtl.ca \\ ilian.bonev@etsmtl.ca
}

\begin{abstract}
In this paper, the self motions of a novel 3-DOF fully decoupled translational parallel robot, called the Pantopteron, are presented. The Pantopteron is similar to the well-known Cartesian parallel manipulator (Tripteron), but due to the use of pantograph linkages, an amplification effect is achieved. Therefore, equipped with the same actuators, the mobile platform of the Pantopteron moves faster than that of the Tripteron. This amplification is defined by the magnification factor of the pantograph linkages. The self motions are probably the most critical types of singularities a manipulator can meet. Therefore it is of utmost importance to have a good knowledge of them. Design considerations are also discussed in order to create Pantopterons without self motions.
\end{abstract}

\section{INTRODUCTION}

From an industrial point of view, the complexity and the existence of numerous singular configurations seem to be the worse drawback of parallel robots because these configurations reduce the workspace, which is already smaller than that of similarly-sized serial robots. Fortunately, the determination of singularities is a well studied problem and several methods have already been presented [1-3].

The worst singular configuration a parallel robot can meet is the Type 2 singularity [1]. In such a singularity, the robot gains at least one degree of freedom and cannot resist some wrenches applied to its platform. Furthermore, the robot cannot exit such a singular configuration, without external help. Type 2 singular configurations can be divided into two classes, depending on the nature of the degree(s) of freedom gained, being either infinitesimal or finite, i.e., self-motion. However, merely studying the Jacobian matrix of the robot [1,2], one cannot identify the nature of Type 2 singularities.

"Corresponding author, ph.: +1-514-396-8403, e-mail: ilian.bonev@etsmtl.ca.
Symmetry and, more precisely, design conditions that simplify the generally too complex direct kinematics of parallel robots are often privileged by robot designers. Unfortunately, such design conditions usually lead to self-motions, which are certainly the worst type of singularity. Hence, it is essential that the design conditions for such self-motions be well known in order to be avoided.

Several papers discuss self-motions in parallel robots. Not surprisingly, most of them deal with the Gough-Stewart platform whose direct kinematic model leads to as many as forty real solutions, for a relatively general design. Design conditions simplifying the direct kinematics of the Gough-Stewart platform, and subsequently leading to self-motions, are given in [4-8]. A classification of all self-motions of the StewartGough platform is presented in [9]. It is shown that the selfmotions can be translations, pure rotations, generalized screw motions, motions equivalent to the displacements of spherical four-bar mechanisms, or more complex spatial motions.

The Stewart-Gough platform is not the only parallel robot with self-motions. A few other parallel robots having selfmotions have also been studied. For example, in [10], it is shown that all singularities of the special 3- $\underline{R} R R$ ( $R$ stands for a passive revolute joint and $\underline{R}$ for an actuated revolute joint) spherical parallel robot, known as the Agile Eye, are selfmotions. The analysis of self mobility of spatial $5 R$ closedloop mechanisms with one degree of freedom are presented in [11]. Reference [12] discusses the determination of generalized analytical expressions for the analysis of self-motions and presents several examples for both planar and spatial mechanisms with legs composed of $R$ joints. The self motions of planar 3- $\underline{R} P R$ manipulators are also studied in $[13,14]$.

In this paper, we will study the self-motions of the so-called Pantopteron [15]. The Pantopteron is a manipulator similar to the Cartesian parallel manipulator (Tripteron) [16], but due to 


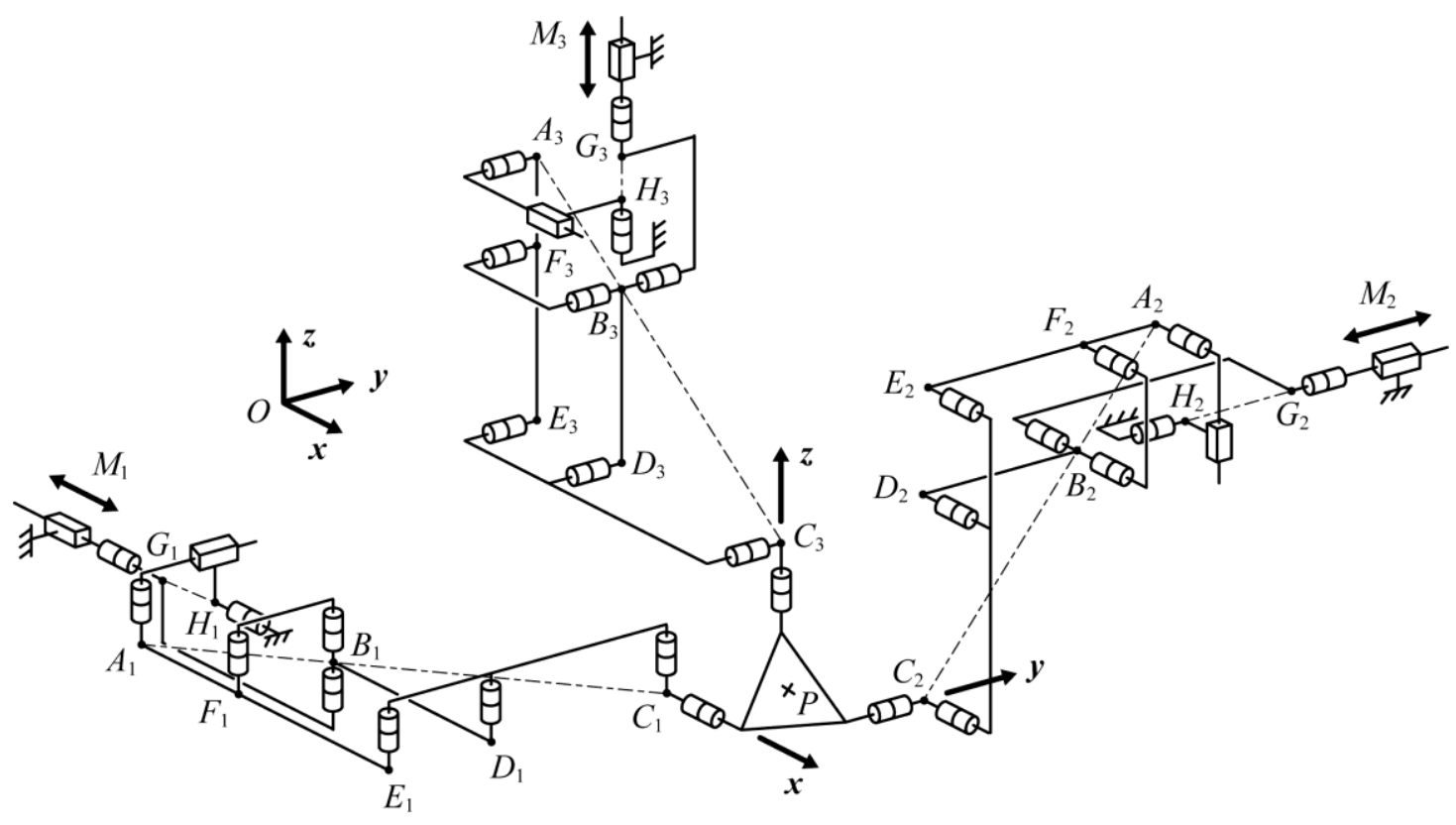

Figure 1. Schematics of the Pantopteron

the use of three pantograph linkages, an amplification of the actuators displacements is achieved. Therefore, given the same actuators, the mobile platform of the Pantopteron moves faster than that of the Tripteron. This amplification is equal to the magnification factor of the pantograph linkages.

The paper is organized as follows. Next, the architecture of the Pantopteron is briefly recalled. Then, a constraint analysis is presented, which will lead to the determination of the self motions. These singularities are kinematically described and various design considerations for the avoidance of self motions are given. Finally, conclusions are drawn.

\section{DESCRIPTION OF THE ARCHITECTURE}

The architecture of the Pantopteron is schematized in Fig. 1. It is composed of three identical legs which correspond to pantograph linkages (Fig. 2).

The pantograph is a mechanical system with two input points, $A_{i}$ and $B_{i}$, and one output point $C_{i}$ (in the remainder of this paper, $i=1,2,3)$. These input points linearly control the displacement of the output point $C_{i}$. A kinematic analysis shows that a linear actuator connected with input point $B_{i}$ controls the vertical displacement of the output point $C_{i}$ and another linear actuator with an axis parallel to $\mathbf{a}_{1 i}$ controls the displacements along the same axis. Note that these motions are completely decoupled, i.e., they can be carried out independently. The input-output relationships for displacements are linear and are determined by the magnification factor $k$ of the pantograph $\left(k=A_{i} C_{i} / A_{i} B_{i}\right)$. These properties of the pantograph mechanism are used in the Pantopteron

For the Pantopteron, the actuators are located at the linear joints (1i) (Fig. 2). These three pairs are connected to the base so that their axes are orthogonal. All other joints are passive. Each pantograph linkage is attached to the platform at point $C_{i}$ via a Cardan joint, the axes each joint (12i) being orthogonal.

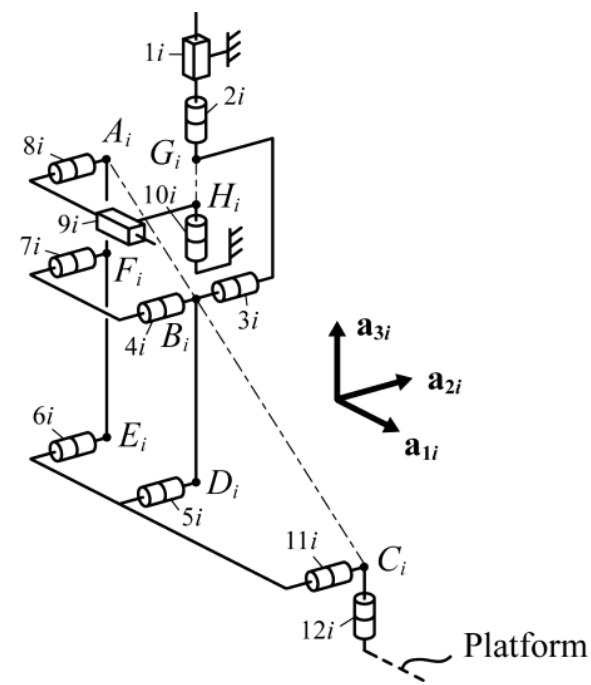

Figure 2. Schematics of a leg of the Pantopteron

They also are connected to actuator (1i) via a revolute joint, which allows the leg to have five DOFs, three translations and two rotations about the axes of the Cardan joint located at $C_{i}$. Such an architecture allows three fully-decoupled translational DOFs and its kinematics can be written under the form:

$$
\dot{\mathbf{x}}=\mathbf{J} \dot{\mathbf{q}},
$$

where

$$
\mathbf{J}=\left[\begin{array}{lll}
k & 0 & 0 \\
0 & k & 0 \\
0 & 0 & k
\end{array}\right] .
$$


$k$ is the magnification factor of the pantograph linkages, $\dot{\mathbf{x}}$ is the vector of the platform velocities and $\dot{\mathbf{q}}$ the vector of the actuator velocities (see [15] for further details).

\section{CONSTRAINTS ANALYSIS}

\subsection{Constraints applied on the platform}

Let $\boldsymbol{x}, \boldsymbol{y}, \boldsymbol{z}$ be the axes of the base frame (Fig. 1). It can be shown (see [15]) that each leg applies two wrenches on the platform:

- a moment $\mathbf{r}_{1 i}(i=1,2,3)$, which is orthogonal to the twist of all pairs (when actuators are disconnected);

- a force $\mathbf{r}_{2 i}$ which is due to the actuator $M_{i}$.

Let us consider leg 1 . We denote by $\mathbf{e}_{j}(j=1$ to 5$)$ the unit screw corresponding to the passive displacement of the platform. The expression of these screws, expressed in the base frame at point $C_{1}$, can be written as:

- for the translations along $\boldsymbol{x}, \boldsymbol{y}$ and $z$, $\mathbf{e}_{11}=\left[\begin{array}{llllll}0 & 0 & 0 & 1 & 0 & 0\end{array}\right]^{T}, \quad \mathbf{e}_{21}=\left[\begin{array}{llllll}0 & 0 & 0 & 0 & 1 & 0\end{array}\right]^{T}$ and $\mathbf{e}_{31}=\left[\begin{array}{llllll}0 & 0 & 0 & 0 & 0 & 1\end{array}\right]^{T}$;

- for the rotations about the axes of joints $(12,1)$ and $(11,1)$, $\mathbf{e}_{41}=\left[\begin{array}{llllll}\cos \gamma_{1} & \cos \theta_{1} \sin \gamma_{1} & \sin \theta_{1} \sin \gamma_{1} & 0 & 0 & 0\end{array}\right]^{T}$ and $\mathbf{e}_{51}=\left[\begin{array}{llllll}0 & -\sin \theta_{1} & \cos \theta_{1} & 0 & 0 & 0\end{array}\right]^{T}$, where $\theta_{1}$ is the angle between $\mathbf{a}_{11}$ and $\boldsymbol{y}$ axes, and $\gamma_{1}$ represents the rotation between vector $\mathbf{a}_{31}$ and the axis of joint $(12,1)$.

The Plücker coordinates of the unit screws can be described in matrix $\mathbf{E}_{\mathbf{1}}$ as

$\mathbf{E}_{\mathbf{1}}=\left[\begin{array}{c}\mathbf{e}_{11}^{T} \\ \mathbf{e}_{21}^{T} \\ \mathbf{e}_{31}^{T} \\ \mathbf{e}_{41}^{T} \\ \mathbf{e}_{51}^{T}\end{array}\right]=\left[\begin{array}{cccccc}0 & 0 & 0 & 1 & 0 & 0 \\ 0 & 0 & 0 & 0 & 1 & 0 \\ 0 & 0 & 0 & 0 & 0 & 1 \\ \cos \gamma_{1} & \cos \theta_{1} \sin \gamma_{1} & \sin \theta_{1} \sin \gamma_{1} & 0 & 0 & 0 \\ 0 & -\sin \theta_{1} & \cos \theta_{1} & 0 & 0 & 0\end{array}\right]$

The wrench $\mathbf{r}_{11}$, transmitted to the platform by the leg, is orthogonal to the twists composing the lines of matrix $\mathbf{E}_{\mathbf{1}}$ :

$$
\mathbf{r}_{11}=\left[\begin{array}{llllll}
r_{11}^{x} & r_{11}^{y} & r_{11}^{z} & 0 & 0 & 0
\end{array}\right]^{T}
$$

with

$$
\begin{aligned}
& r_{11}^{x}=-\sin \gamma_{1} \\
& r_{11}^{y}=\cos \theta_{1} \cos \gamma_{1} \\
& r_{11}^{z}=\sin \theta_{1} \cos \gamma_{1}
\end{aligned}
$$

Thus, $\mathbf{r}_{11}$ is a wrench of zero pitch (a pure moment).

Similarly, it is possible to find that the wrenches $\mathbf{r}_{1 i}$ transmitted to the platform by the legs when all actuators are disconnected are all pure moments. Let $\mathbf{Q}$ be the matrix composed of these wrenches applied on the platform by the legs. The expression of $\mathbf{Q}$ in the base frame, and expressed at point $O$, is:

$$
\mathbf{Q}=\left[\begin{array}{l}
\mathbf{r}_{11}^{T} \\
\mathbf{r}_{12}^{T} \\
\mathbf{r}_{13}^{T}
\end{array}\right]=\left[\begin{array}{llllll}
r_{11}^{x} & r_{11}^{y} & r_{11}^{z} & 0 & 0 & 0 \\
r_{12}^{x} & r_{12}^{y} & r_{12}^{z} & 0 & 0 & 0 \\
r_{13}^{x} & r_{13}^{y} & r_{13}^{z} & 0 & 0 & 0
\end{array}\right]
$$

with

$$
\begin{aligned}
r_{12}^{x} & =\sin \theta_{2} \cos \gamma_{2} \\
r_{12}^{y} & =-\sin \gamma_{2} \\
r_{12}^{z} & =\cos \theta_{2} \cos \gamma_{2} \\
r_{13}^{x} & =\cos \theta_{3} \cos \gamma_{3} \\
r_{13}^{y} & =\sin \theta_{3} \cos \gamma_{3} \\
r_{13}^{z} & =-\sin \gamma_{3}
\end{aligned}
$$$$
\text { and }
$$

The passive displacements of the platform are orthogonal to this matrix of rank equal to 3 . In the general case, there are three independent passive displacements, which are the three translations about $\boldsymbol{x}, \boldsymbol{y}$ and $z$ axes. Thus, the platform is constrained by the legs to have only translational displacements.

As joints $(12, i)$ are all orthogonal to each other, the following relations must hold:

$$
\begin{aligned}
\mathbf{e}_{41}^{T} \mathbf{e}_{42} & =0 \\
\mathbf{e}_{\mathbf{4 2}}^{T} \mathbf{e}_{43} & =0 \\
\mathbf{e}_{41}^{T} \mathbf{e}_{43} & =0 \\
\text { with } & \\
\mathbf{e}_{\mathbf{4 1}} & =\left[\begin{array}{lllllll}
\cos \gamma_{1} & \cos \theta_{1} \sin \gamma_{1} & \sin \theta_{1} \sin \gamma_{1} & 0 & 0 & 0
\end{array}\right]^{T} \\
\mathbf{e}_{42} & =\left[\begin{array}{lllllll}
\sin \theta_{2} \sin \gamma_{2} & \cos \gamma_{2} & \cos \theta_{2} \sin \gamma_{2} & 0 & 0 & 0
\end{array}\right]^{T} \\
\mathbf{e}_{43} & =\left[\begin{array}{lllllll}
\cos \theta_{3} \sin \gamma_{3} & \sin \theta_{3} \sin \gamma_{3} & \cos \gamma_{3} & 0 & 0 & 0
\end{array}\right]^{T}
\end{aligned}
$$

Developing and simplifying Eqs. (9) leads to:

$$
\begin{aligned}
f_{1}= & \cos \gamma_{1} \sin \theta_{2} \sin \gamma_{2}+\cos \theta_{1} \sin \gamma_{1} \cos \gamma_{2} \\
& +\sin \theta_{1} \sin \gamma_{1} \cos \theta_{2} \sin \gamma_{2}=0 \\
f_{2}= & \cos \gamma_{2} \sin \theta_{3} \sin \gamma_{3}+\cos \theta_{2} \sin \gamma_{2} \cos \gamma_{3} \\
& +\sin \theta_{2} \sin \gamma_{2} \cos \theta_{3} \sin \gamma_{3}=0 \\
f_{3}= & \cos \gamma_{1} \sin \theta_{3} \sin \gamma_{3}+\cos \theta_{1} \sin \gamma_{1} \cos \gamma_{3} \\
& +\sin \theta_{1} \sin \gamma_{1} \cos \theta_{3} \sin \gamma_{3}
\end{aligned}
$$

Thus it is possible to find the expression of angles $\gamma_{i}$ as a function of angles $\theta_{i}$. Two distinct sets of solutions can be found:

$$
\begin{aligned}
& \gamma_{1}=\gamma_{2}=\gamma_{3}=0 \\
& \gamma_{1}=\tan ^{-1}\left(\frac{\cos \theta_{1} \cos \theta_{2} \cos \theta_{3}+\sin \theta_{1} \sin \theta_{2} \sin \theta_{3}}{-\cos \theta_{2} \sin \theta_{3}+\sin \theta_{1} \sin \theta_{2} \cos \theta_{1} \cos \theta_{3}}\right) \\
& \gamma_{2}=\tan ^{-1}\left(\frac{\cos \theta_{1} \cos \theta_{2} \cos \theta_{3}+\sin \theta_{1} \sin \theta_{2} \sin \theta_{3}}{-\cos \theta_{3} \sin \theta_{1}+\sin \theta_{2} \sin \theta_{3} \cos \theta_{1} \cos \theta_{2}}\right) \\
& \gamma_{3}=\tan ^{-1}\left(\frac{\cos \theta_{1} \cos \theta_{2} \cos \theta_{3}+\sin \theta_{1} \sin \theta_{2} \sin \theta_{3}}{-\cos \theta_{1} \sin \theta_{2}+\sin \theta_{1} \sin \theta_{3} \cos \theta_{2} \cos \theta_{3}}\right)
\end{aligned}
$$$$
\text { or }
$$

These two sets of solutions depend on the assembly of the mechanism. In a practical way, all angles $\gamma_{i}$ are set to $0^{\circ}$. 
However, it will be seen later that the self motions of the mechanisms can be found by the analysis of Eqs. (13).

Let us now consider that the actuator $M_{1}$ located at joint $(1,1)$ is fixed. So, due to the decoupling properties of the pantograph linkages, the position about $\boldsymbol{x}$ axis of point $C_{1}$ is fixed. Thus, the platform has now two passive translational DOFs, which are orthogonal to the $\boldsymbol{x}$ axis. Therefore, a supplementary constraint is applied on the platform, which restrains its displacement.

Using an approach similar to the previous one, the second wrench applied by the leg on the platform, expressed at point $C_{1}$, is $\mathbf{r}_{21}=\left[\begin{array}{llllll}0 & 0 & 0 & 1 & 0 & 0\end{array}\right]^{T}$.

Let us denote by $\mathbf{R}$ the matrix composed of these wrenches applied on the platform by the legs. The expression of $\mathbf{R}$ in the base frame, and expressed at point $O$, is:

$$
\mathbf{R}=\left[\begin{array}{l}
\mathbf{r}_{11}^{T} \\
\mathbf{r}_{21}^{T} \\
\mathbf{r}_{12}^{T} \\
\mathbf{r}_{22}^{T} \\
\mathbf{r}_{13}^{T} \\
\mathbf{r}_{23}^{T}
\end{array}\right]=\left[\begin{array}{cccccc}
0 & \cos \theta_{1} & \sin \theta_{1} & 0 & 0 & 0 \\
0 & z_{C 1} & -y_{C 1} & 1 & 0 & 0 \\
\sin \theta_{2} & 0 & \cos \theta_{2} & 0 & 0 & 0 \\
-z_{C 2} & 0 & x_{C 2} & 0 & 1 & 0 \\
\cos \theta_{3} & \sin \theta_{3} & 0 & 0 & 0 & 0 \\
-x_{C 3} & y_{C 3} & 0 & 0 & 0 & 1
\end{array}\right]
$$

where $x_{C i}, y_{C i}$ and $z_{C i}$ are the expressions of the coordinates of points $C_{i}$ along $\boldsymbol{x}, \boldsymbol{y}$ and $z$ axes.

\subsection{Degeneracy of constraints}

As the mechanism is fully isotropic, it does not have any Type 2 singularities. However, it has been shown in [15] that it has constraint singularities [17]. These singular configurations appear if the system of wrenches applied on the platform degenerates, i.e., if the matrix $\mathbf{R}$ of Eq. (14) becomes singular. Such a singularity is called a constraint singularity. This happens if:

$$
\operatorname{det}(\mathbf{R})=\cos \theta_{1} \cos \theta_{2} \cos \theta_{3}+\sin \theta_{1} \sin \theta_{2} \sin \theta_{3}=0
$$

Introducing this expression in Eqs. (13), it can be seen that, in a constraint singularity, all angles $\gamma_{i}$ may admit only one solution (indeed a double solution), which is $\gamma_{i}=0$. This is true if the denominators of the Eqs. (13) are different from 0 . If not, expressions (13) are indefinite, which implies that the system of Eqs. (11) degenerates.

Thus, let us analyze the degeneracy of Eqs. (13). These solutions degenerate if the following system of equations admits solutions:

$$
\begin{aligned}
& h_{1}=\cos \theta_{1} \cos \theta_{2} \cos \theta_{3}+\sin \theta_{1} \sin \theta_{2} \sin \theta_{3}=0 \\
& h_{2}=-\cos \theta_{2} \sin \theta_{3}+\sin \theta_{1} \sin \theta_{2} \cos \theta_{1} \cos \theta_{3}=0 \\
& h_{3}=-\cos \theta_{3} \sin \theta_{1}+\sin \theta_{2} \sin \theta_{3} \cos \theta_{1} \cos \theta_{2}=0 \\
& h_{4}=-\cos \theta_{1} \sin \theta_{2}+\sin \theta_{1} \sin \theta_{3} \cos \theta_{2} \cos \theta_{3}=0
\end{aligned}
$$

It can be shown that this system of equations has several sets of solutions, which are:

$$
\begin{aligned}
& \text { or } \theta_{1}=0+n \pi \text { and } \theta_{2}= \pm \pi / 2, n=0,1,2 \ldots \\
& \qquad \theta_{2}=0+n \pi \text { and } \theta_{3}= \pm \pi / 2, n=0,1,2 \ldots \\
& \text { or } \theta_{3}=0+n \pi \text { and } \theta_{1}= \pm \pi / 2, n=0,1,2 \ldots
\end{aligned}
$$

Let us consider the case of Eq. (17a) (Fig. 3). Introducing these new conditions into the system of Eqs. (11) leads to the following set of solutions:

$$
\gamma_{3}=0 \text { and } \gamma_{1}=-\gamma_{2}+n \pi, n=0,1,2 \ldots
$$

Thus, in such a case, the values of angles $\gamma_{1}$ and $\gamma_{2}$ are inde-

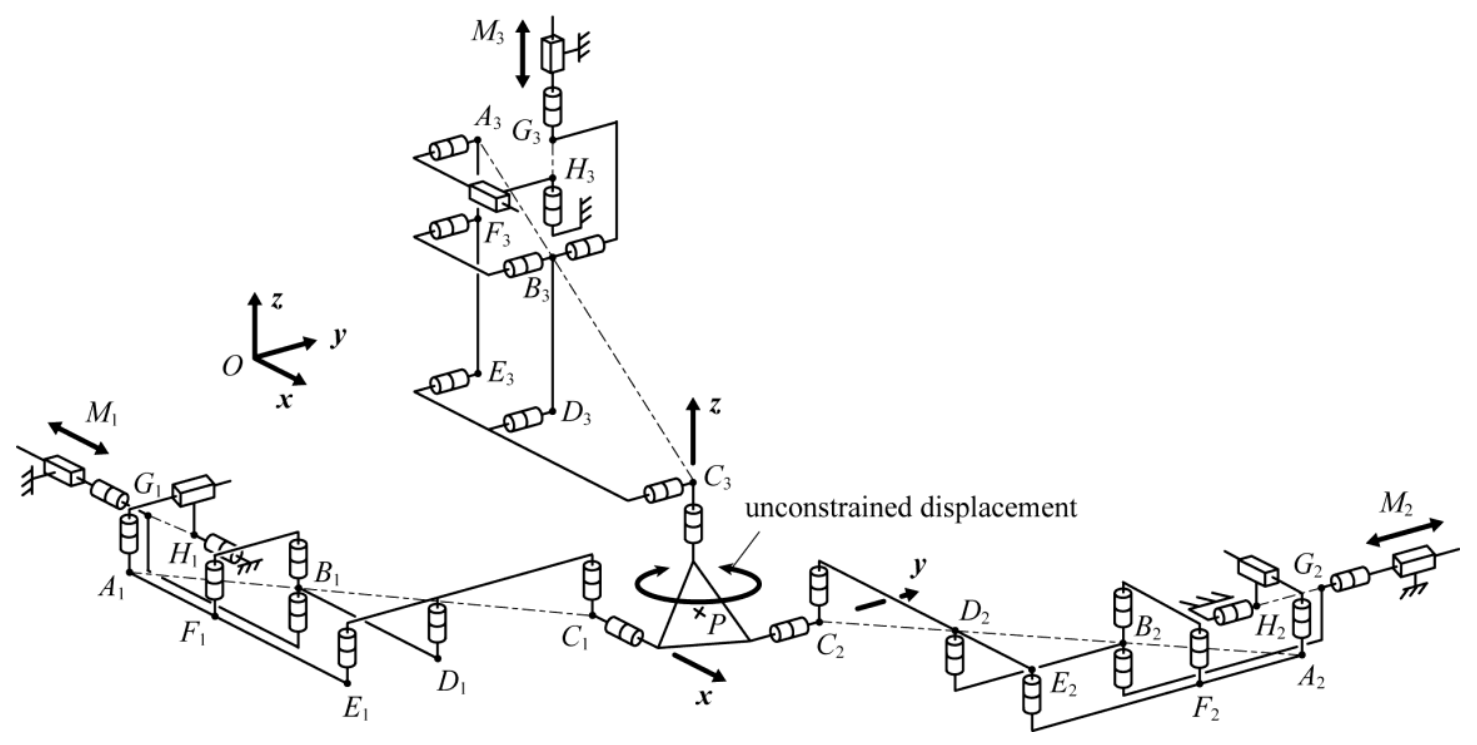

Figure 3. Example of a constraint singularity for $\theta_{1}=0$ and $\theta_{2}=90^{\circ}$. 
terminate. Note that the same solutions for $\gamma_{2}$ and $\gamma_{3}$ or $\gamma_{1}$ and $\gamma_{3}$ could be found by using Eqs. (17b) and (17c), respectively.

In the next part, we will analyze the kinematics of the manipulator in such singular configurations.

\section{SELF MOTION ANALYSIS}

\subsection{Kinematic analysis}

Let us examine the manipulator when $\theta_{1}=0$ and $\theta_{2}=\pi / 2$ (Fig. 3). In such a case, vectors $\mathbf{a}_{2 i}$ of legs 1 and 2 of the manipulator are collinear, i.e., the planes of the pantograph linkages are parallel (here, they are horizontal). From Eq. (18), $\gamma_{3}$ $=0$, therefore the altitude of the platform (and consequently of points $C_{1}$ and $C_{2}$ of the pantograph linkages, so as the values of angles $\theta_{1}$ and $\theta_{1}$ ) cannot change if actuator $M_{3}$ is fixed.

As the planes of the pantograph linkages stay horizontal, the passive prismatic joints of legs 1 and 2 are also horizontal plane (but also perpendicular), which implies that point $C_{1}$ can have a passive translation along the $\boldsymbol{y}$ axis, and point $C_{2}$ a passive translation along the $\boldsymbol{x}$ axis. Moreover, as the rotation $\theta_{3}$ of joints $(2,3)$ and $(10,3)$ of leg 3 , such as the displacement of the prismatic joint $(9,3)$, are not constrained (these joints are passive), the planar displacement of point $C_{3}$ of the platform is completely free.

Figure 4 represents the planar view of the manipulator in such a singularity. For clarity, leg 3 is replaced by an equivalent passive $R P$ chain which completely allows the same planar displacement as the pantograph linkage.

As points $C_{1}$ and $C_{2}$ of the platform are constrained to move along lines $\mathcal{L}_{1}$ and $\mathcal{L}_{2}$, respectively, and as the planar displacement of point $C_{3}$ is free, the platform undergoes a socalled Cardanic (self) motion [13,14,18,19].

As a consequence, each point $Q$ of the platform describes a curve $\mathcal{E}(Q)$, which may be:

- a straight line;

- a doubly traced segment;

- $\quad$ an ellipse.

The same type of self motions will be found by analyzing the conditions (17b) and (17c), but the movements will take place in other planes.

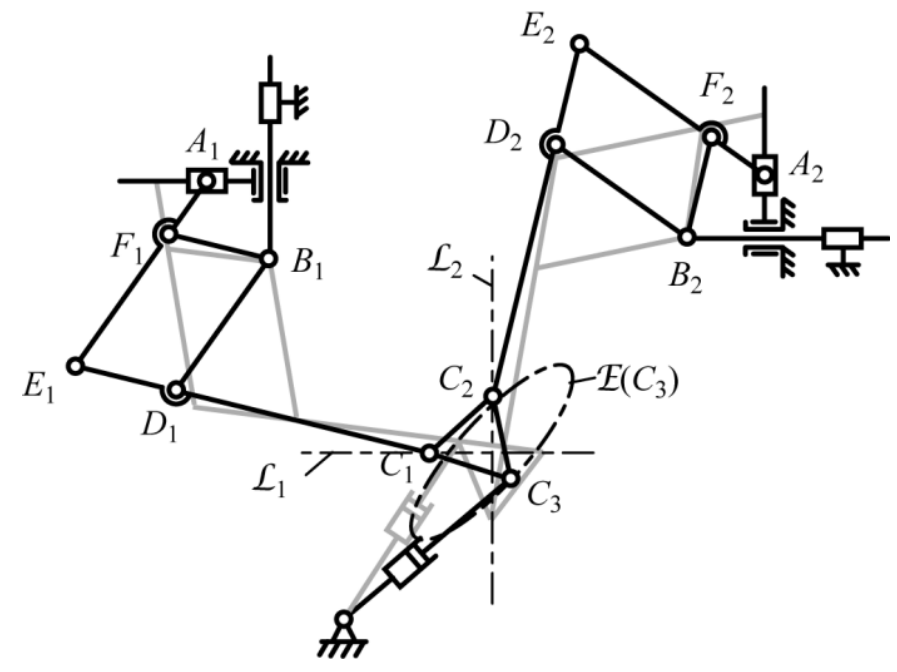

Figure 4. Cardanic self motion of the Pantopteron
In the next section, we will analyze the conditions on the design parameters that allow creating mechanisms without self motions.

\subsection{Design considerations}

Let us analyze once again the Eq. (17a), which can also be written under the form:

$\sin \theta_{1}=0$ and $\cos \theta_{2}=0$.

In the Cartesian space, Eq. (19) can be expressed as:

$$
\frac{\left(z_{C 1}-z_{G 1}\right)}{\Gamma_{1}}=0 \text { and } \frac{\left(z_{C 2}-z_{G 2}\right)}{\Gamma_{2}}=0
$$

where

$$
\begin{aligned}
& \Gamma_{1}=\sqrt{\left(y_{C 1}-y_{G 1}\right)^{2}+\left(z_{C 1}-z_{G 1}\right)^{2}} \\
& \Gamma_{2}=\sqrt{\left(z_{C 2}-z_{G 2}\right)^{2}+\left(x_{C 2}-x_{G 2}\right)^{2}}
\end{aligned}
$$

and $x_{C i}, y_{C i}$ and $z_{C i}\left(x_{G i}, y_{G i}\right.$ and $z_{G i}$, resp.) are the expressions of the coordinates of points $C_{i}\left(G_{i}\right.$, resp.) along $\boldsymbol{x}, \boldsymbol{y}$ and $\boldsymbol{z}$ axes.

Disregarding the case where $\Gamma_{i}$ tends to infinity, singularities appear when:

$$
z_{C 1}-z_{G 1}=0 \text { and } z_{C 2}-z_{G 2}=0
$$

Taking into account that the terms $x_{G i}, y_{G i}, z_{G i}$ appearing in Eq. (22) are constant and that

$$
\mathbf{O C}_{i}=[x, y, z]^{T}+\left[a_{i}, b_{i}, c_{i}\right]^{T}
$$

where $a_{i}, b_{i}, c_{i}$ are constant terms, Eq. (22) can be rewritten under the form:

$$
z+c_{1}-z_{G 1}=z+c_{2}-z_{G 2}=0
$$

Thus, condition (17a) can appear if:

$$
c_{1}-z_{G 1}=c_{2}-z_{G 2} \text {. }
$$

By a similar way, it can be proven that condition (17b) may appear if:

$$
a_{1}-x_{G 1}=a_{2}-x_{G 2},
$$

and condition $(17 \mathrm{c})$ if:

$$
b_{1}-y_{G 1}=b_{2}-y_{G 2} \text {. }
$$

Thus, by avoiding these three conditions, the mechanism will never have self motions.

\section{CONCLUSIONS}

The singularities of a novel 3-DOF fully-decoupled isotropic translational parallel mechanism, called the Pantopteron, were analyzed in detail. It was shown that the only sin- 
gularities that this robot can undergo are so-called constraint singularities. Furthermore, all of these constraint singularities were shown to be Cardanic self-motions. Fortunately, simple design conditions for eliminating the possibility for such singularities were found.

\section{REFERENCES}

[1] Gosselin, C.M., and Angeles, J., 1990, "Singularity Analysis of Closed-Loop Kinematic Chains," IEEE Transactions on Robotics and Automation, Vol. 6, No. 3, pp. 331-336.

[2] Bonev, I.A., Zlatanov, D., and Gosselin, C.M., 2003, "Singularity Analysis of 3-DOF Planar Parallel Mechanisms via Screw Theory," Journal of Mechanical Design, Vol. 125, No. 3, pp. 573-581.

[3] Zlatanov, D., Fenton R.G., and Benhabib B., 1994, "Singularity Analysis of Mechanisms and Robots via a Velocity-Equation Model of the Instantaneous Kinematics," IEEE International Conference on Robotics and Automation, San Diego, CA, USA, pp. 980-991.

[4] Karger, A., 2001, "Singularities and Self-motions of Equiform Platforms," Mechanism and Machine Theory, Vol. 36, pp. 801-815.

[5] Karger, A., 2003, "Architecture Singular Planar Parallel Robots," Mechanism and Machine Theory, Vol. 38, No. 11, pp. 1149-1164.

[6] Husty, M.L., and Zsombor-Murray, P., 1994, "A Special Type of Singular Stewart-Gough Platform," Advances in Robot Kinematics and Computational Geometry, J. Lenarcic and B. Ravani (eds.), Kluwer Academic Publishers, pp. 449-458.

[7] Husty, M.L., and Karger, A., 2000, "Self-Motions of Griffis-Duffy Type Parallel Robots," IEEE International Conference on Robotic and Automation, San Francisco, CA, USA, pp. 7-12.

[8] Wohlhart, K., 2003, "Mobile 6-SPS Parallel Robots," Journal of Robotic Systems, Vol. 20, No. 8, pp. 509-516.

[9] Karger, A., and Husty, M.L., 1998, "Classification of all Self-Motions of the Original Stewart-Gough Platform," Computer-Aided Design, Vol. 30, No. 3, pp. 205-215.
[10] Bonev, I.A., Chablat, D., and Wenger, P., 2006, "Working and Assembly Modes of the Agile Eye," IEEE International Conference on Robotics and Automation, Orlando, FL, USA, pp. 2317-2322.

[11] Karger, A., 1998, "Classification of 5R Closed Kinematic Chain with Self Mobility," Mechanism and Machine Theory, Vol. 33, No. 1, pp. 213-222.

[12] Bandyopadhyay, S., and Ghosal, A., 2004, "Analysis of Configuration Space Singularities of Closed-Loop Mechanisms and Parallel Robots," Mechanism and Machine Theory, Vol. 39, No. 5, pp. 519-544.

[13] Chablat, D., Wenger, P. and Bonev, I.A., 2006, "SelfMotions of a Special 3-RPR Planar Parallel Robot," Advances in Robot Kinematics, J. Lenarcic and B. Roth (eds.), Springer, pp. 221-228.

[14] Briot, S., Bonev, I.A., Chablat, D., Wenger, P. and Arakelian, V., 2008, "Self-Motions of General 3- $\underline{R} P R$ Planar Parallel Robots," International Journal of Robotics Research, Vol. 27, No. 7, pp. 855-866.

[15]Briot, S. and Bonev, I.A., "Pantopteron: a New FullyDecoupled 3-DOF Translational Parallel Robot for Pickand-Place Applications," ASME Journal of Mechanisms and Robotics, Vol. 1, No. 2, 2009.

[16] Gosselin, C.M., and Kong, X., 2004, "Cartesian Parallel Manipulators," US patent 6,729,202, filed July 8, 2002, and issued May 4, 2004.

[17]Zlatanov, D., Bonev, I. A., and Gosselin, C.M., 2002, "Constraint Singularities of Parallel Mechanisms," IEEE International Conference on Robotics and Automation (ICRA 2002), Washington, D.C., USA, May 11-15.

[18] Sekulie, A., 1998, "Method of Synthesis of Cardanic motion," Facta Universitatis, Mechanical Engineering, University of NIS, Vol. 1, No. 5, pp. 565-572.

[19] Tischler, C.R., Hunt, K.H. and Samuel, A.E., 1998, “A Spatial Extension of Cardanic Movement: Its Geometry and some Derived Mechanisms," Mechanism and Machine Theory, Vol. 33, No. 8, pp. 1249-1276. 